\title{
AVALIAÇÃO OVARIANA MORFOMÉTRICA E RECUPERAÇÃO DE COMPLEXOS Cumulus oophorus DE CADELAS EM DIFERENTESS FASES DO CICLO ESTRAL
}

\author{
Luiz Carlos Binsfeld ${ }^{1}$, Ramiro Vasconcelos Darriba², Jessé Lahos Borges², Luciana \\ Kazue Otutumi², Ivo Walter dos Santos ${ }^{1}$, Luciana da Silva Leal ${ }^{3}$ \\ 1 UFPR \\ 2 UNIPAR \\ 3 UEPG \\ Correspondência: Luiz Carlos Binsfeld: binsfeld@ufpr.br
}

\begin{abstract}
RESUMO: A maturação in vitro de oócitos caninos ainda apresenta resultados insatisfatórios, devido às particularidades da fisiologia reprodutiva da cadela, incluindo as diferentes fases do ciclo estral que interferem nos resultados. Os objetivos do presente trabalho foram avaliar a morfometria ovariana e identificar a influência da fase do ciclo estral sobre a quantidade e a qualidade dos complexos Cumulus oophorus (CCOs) recuperados de cadelas. Ao todo, foram avaliados os ovários de 55 cadelas submetidas à técnica de ovário-salpingo-histerectomia eletiva. Para as mensurações das dimensões e peso dos ovários foram utilizados paquímetro e balança digital. Para a avaliação da concentração plasmática de progesterona se empregou a técnica de radioimunoensaio. A recuperação dos CCOs foi procedida por fatiamento e escarificação dos ovários, enquanto que a classificação dos mesmos foi realizada por avaliação em estereomicroscópios (aumento 40x). Com relação à avaliação morfométrica dos ovários, $100 \%$ apresentaram coloração rósea; $96,36 \%$ formato oval e 3,64\% formato redondo; 94,55\% tinham consistência fibroelástica e 5,45\% consistência flácida. O peso médio dos ovários \pm erro padrão foi de $0,58 \pm 0,08 \mathrm{~g}$. Já as medidas médias \pm erro padrão de comprimento, largura e altura foram, respectivamente, de: $13,41 \pm 0,50 ; 7,81 \pm 0,31$ e 6,46 $\pm 0,27 \mathrm{~mm}$. O número médio de corpos lúteos identificados foi de $3,62 \pm 0,49$. No total foram recuperados 1642 CCOs, cuja média e erro padrão/animal foram de 29,85 $\pm 4,20$; dentre estes $58,16 \%$ foram classificados como grau $1 ; 26,19 \%$ grau $2 ; 3,78 \%$ grau 3 e $11,88 \%$ expandido. Conclui-se que a fase do ciclo estral não influenciou no número total e na qualidade dos CCOs.

Palavras-chave: ciclo estral; oócito; ovário; progesterona
\end{abstract}

\section{OVARIAN MORPHOMETRIC EVALUATION AND RECOVERY OF CUMULUS- OOCYTE COMPLEXES OF BITCHES IN DIFFERENT PHASES OF ESTROUS CYCLE}

\begin{abstract}
In vitro maturation of canine oocyte still has unsatisfactory results due to the peculiarities of the reproductive physiology of the female dog, including the different phases of the estrous cycle that interfere with the results. The aims of this study were to evaluate ovarian morphology and identify the influence of estrous cycle on the quantity and quality of Cumulus-oocyte complexes (COCs) recovered from bitches. In total, were evaluated 55 ovaries of bitches submitted to elective surgery for ovariohysterectomy. For measurements of ovarian size and weight was used digital paquimeter and balance. To evaluate the plasma progesterone concentration was employed the radioimmunoassay. The recovery of COCs was accomplished by slicing and scarification of ovary, while classifying of COCs was performed by stereomicroscopes (40x). About morphometric study of ovaries, $100 \%$ were pinkish; $96.36 \%$ had oval and $3.64 \%$ round shape, $94.55 \%$ had fibroelastic and $5.45 \%$ had flaccid consistency. The average weight \pm standard error of the ovaries was $0.58 \pm 0.08 \mathrm{~g}$. The mean measurements \pm standard error of length, width and height were, respectively: $13.41 \pm 0.50,7.81 \pm 0.31$ and $6.46 \pm 0.27 \mathrm{~mm}$. The average number of corpus luteum was identified was $3.62 \pm 0.49$. A total of 1642 COCs were recovered, with a mean and standard deviation/animal were $29.85 \pm 4.20$; among them $58.16 \%$ were classified as grade $1,26.19 \%$ grade $2,3.78 \%$ grade 3 and $11.88 \%$ expanded. It can be concluded that the estrous cycle did not influence the total number and quality of COCs.
\end{abstract}

Key Words: estrous cycle; oocyte; ovary; progesterone 


\section{INTRODUÇÃO}

Nas últimas décadas tem sido expressivo o crescimento do número de animais de companhia, bem como se observou um estreitamento da relação do homem com seu animal de estimação. Tais situações contribuíram para que a criação de cães de raças se tornasse lucrativa, aumentando 0 interesse na reprodução, principalmente pelos canis. Em consequência, a pesquisa científica ganhou impulso, passando a aprimorar as biotecnologias como inseminação artificial, congelação de sêmen e produção in vitro de embriões.

A fisiologia reprodutiva de canídeos apresenta muitas particularidades, tornando a cadela um modelo reprodutivo único. Entre as especificidades reprodutivas caninas tem-se a duração do período fértil, da ovulação, a maturação e a fecundação dos oócitos (Oliveira; Marques Jr.; Neves, 2003; Luz; Freitas; Pereira, 2005).

Os ovários caninos estão situados dorsalmente na região sublombar, caudalmente aos rins. Apresentam forma elíptica e riniforme, alcançando um comprimento de 1,0 a $1,5 \mathrm{~cm}$ (Dyce; Sack; Wensing, 2004; König, 2004). O córtex ovariano contém folículos e corpos lúteos (CLs) em vários estágios de desenvolvimento e regressão (Hafez; Hafez, 2004), dependendo da fase do ciclo estral.

As fases do ciclo reprodutivo da cadela incluem: proestro, estro, diestro e anestro (Jöchle; Andersen, 1977). O proestro se caracteriza pelo desenvolvimento folicular, aumentando continuamente a concentração sérica de estradiol $\left(E_{2}\right)$ que é sintetizado pelas células da granulosa de tais folículos (Oliveira; Marques Jr., 2006). No entanto, nas cadelas verifica-se a ocorrência de luteinização pré- ovulatória, conferindo níveis crescentes de progesterona $\left(P_{4}\right)$ na fase final do proestro (Concannon; Hansel; Visek, 1975; England, 1998).

O estro constitui a fase de atividade reprodutiva (Jöchle; Andersen, 1977), na qual ocorrem as ovulações (Tsutsui, 1989). O diestro é a etapa de instalação dos CLs que permanecem $\begin{array}{lllll}\text { ativos } e & \text { secretam } & \mathrm{P}_{4} & \text { por }\end{array}$ aproximadamente dois meses (Derivaux, 1980; Concannon; Digregorio, 1986; Musolino; Ghirelli; Moreno, 2000). O anestro estende-se da regressão dos CLs e repouso do aparelho genital até a próxima fase folicular (Derivaux, 1980; Feldman; Nelson, 2004).

Em estudos envolvendo maturação e fertilização in vitro (FIV) na espécie canina, uma das principais preocupações é a quantidade e a qualidade de oócitos recuperados (Rocha et al., 2006). A classificação da qualidade oocitária considera a aparência do citoplasma, que preferencialmente deve ser escuro e homogêneo e a presença de múltiplas camadas de células do Cumulus (Hewitt; England, 1997).

Apesar das mudanças ovarianas e endócrinas características de cada fase, o estágio do ciclo estral parece não influenciar a contagem de oócitos recuperados de cadelas (Rodrigues; Rodrigues, 2003). Por outro lado, a idade e a raça podem alterar a capacidade da fêmea em produzir oócitos viáveis para a utilização em técnicas de FIV (Rocha et al., 2006).

Nesse sentido, em alguns estudos, cadelas jovens apresentaram índices melhores do que as fêmeas mais velhas (Farstad, 2000; Rocha et al., 2006). Rocha et al. (2006) sugeriram que cadelas adultas, com menos de sete anos de idade, possuem um rendimento mais provável de oócitos de 
maior qualidade do que animais muito novos ou muito velhos.

É notável que mais pesquisas devam ser efetivadas para caracterizar padrões que identifiquem uma cadela como doadora de oócitos. Desta forma, os objetivos do presente trabalho foram avaliar a morfometria ovariana e identificar a influência da fase do ciclo estral sobre a quantidade e a qualidade dos complexos Cumulus oophorus (CCOs) recuperados de cadelas.

\section{MATERIAL E MÉTODOS}

Foram colhidas amostras de sangue e ovários de 55 cadelas, submetidas ao procedimento de ováriosalpingo-histerectomia (OSH) no projeto "Controle Populacional de Cães e Gatos", realizado no Hospital Veterinário da Universidade Federal do Paraná (UFPR - Palotina/PR), Clínica Veterinária "Petscão" (Umuarama/PR), Clínica Veterinária Agrounião (Palotina/PR) e também na Campanha de Castração de Cães e Gatos da Universidade Paranaense (UNIPAR Umuarama/PR).

Os proprietários dos animais preencheram um questionário que continha dados de identificação (nome, raça, idade e peso) e perguntas a respeito da vida reprodutiva do animal (último episódio de estro, intervalo entre estros, número de partos e data do último parto).

Segundo a distribuição racial, 37 fêmeas não apresentavam raça definida (SRD) e as demais eram das raças Poodle $(n=6)$, Pinscher $(n=3)$, Teckel, Boxer, Cocker, Pit bull, Labrador, Spitz alemão, Chow chow, Llhasa apso e Akita (um animal de cada raça, respectivamente).

As cadelas apresentaram idade média \pm erro padrão de $37,33 \pm 4,82$ meses (mínimo de 5 e máximo de 144 meses) e peso médio \pm erro padrão de
$9,38 \pm 0,99 \mathrm{~kg}$ (mínimo de 1,40 e máximo de $36,00 \mathrm{~kg}$ ).

Das 55 cadelas estudadas, três estavam no proestro, 23 no estro, cinco no diestro e 24 no anestro.

\section{Colheita e Avaliação Morfométrica dos Ovários}

Os ovários de cada fêmea foram colhidos separadamente, mantidos em béqueres identificados, contendo solução fisiológica aquecida a $36^{\circ} \mathrm{C}$ e transportados ao laboratório de Reprodução Animal da UFPR e da UNIPAR, em caixa isotérmica.

No laboratório, os ovários foram dissecados com auxílio de tesoura, pinça anatômica e bisturi. Após a dissecação, as gônadas foram avaliadas quanto à forma (arredondada ou ovalada); coloração (amarelada ou rosada); peso $(\mathrm{g})$; comprimento $(\mathrm{mm})$, largura $(\mathrm{mm})$ e altura $(\mathrm{mm})$, averiguando-se também o número de folículos ovarianos superficiais e presença de corpos lúteos.

O peso do ovário foi determinado em balanca digital (Modelo BL3200H, Shimadzu ${ }^{\circledR}$ ) e as medidas aferidas por paquímetro digital (Modelo 100.174BL; Digimess $^{\circledR}$ ).

A consistência do ovário foi determinada por palpação manual e classificada em: fibroelástica (mais firme) ou flácida (macia), em analogia aos termos utilizados para a avaliação da consistência testicular (BALL et al., 1983; CHÁCON et al., 1999).

\section{Recuperação e Seleção dos Complexos (CCOs) Cumulus oophorus} Realizadas as avaliações morfométricas, os ovários foram acondicionados em placas de petri 60×16 mm, fatiados (slicing) e escarificados com o auxílio de uma lâmina de bisturi e pinça anatômica e lavados com solução de DPBS 
(Dulbecco Phosphate Buffer Saline Vitroce $\|^{\circledR}$ ). Para recuperação dos CCOs, - lavado foi examinado em estereomicroscópio (Modelo Q714z-2, QUIMIS ${ }^{\circledR}$.

Após a recuperação, os CCOs foram classificados em grau 1, 2, 3 ou expandido, de acordo com o número de camadas de células do Cumulus e o aspecto do citoplasma do oócito (HEWITT; ENGLAND,1997), considerando: Grau 1: pigmentação escura do citoplasma do oócito, com uma ou mais camadas de células do cumulus; Grau 2: pigmentação clara do citoplasma do oócito, com camadas incompletas de células do cumulus; Grau 3: pigmentação pálida do citoplasma do oócito, sem formato definido e sem células do cumulus aderidas e Expandidos: células do cumulus oophorus expandidas.

\section{Colheita de Sangue e Análise Hormonal}

As amostras de sangue foram colhidas pela punção da veia jugular ou cefálica, com auxílio de agulha $30 \times 8$ $\mathrm{mm}$ e depositadas em tubos de colheita de sangue a vácuo heparinizados. $O$ volume obtido foi centrifugado a $1500 \times \mathrm{xg}$ por 15 minutos (Modelo TDL80-2B, Centribio $^{\circledR}$ ) para obtenção do plasma, o qual foi acondicionado em tubo do tipo eppendorf e congelado em freezer a $20^{\circ} \mathrm{C}$.

As amostras foram quantificadas em suas concentrações plasmáticas de $\mathrm{P}_{4}(\mathrm{ng} / \mathrm{mL})$ por meio do procedimento de radioimunoensaio, utilizando-se para tal kit comercial de fase sólida (Coat A Count Diagnostics Products Corporation), no laboratório de Endocrinologia do Departamento de Reprodução Animal e Radiologia Veterinária, FMVZ - UNESP - Campus Botucatu/SP.

A fase do ciclo estral foi determinada segundo a concentração plasmática de $\mathrm{P}_{4}$, usando-se os valores apresentados na tabela 1 .

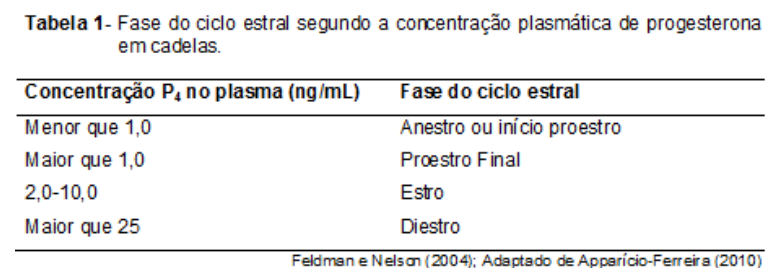

\section{Análise Estatística}

Os resultados obtidos foram analisados por meio de estatística descritiva (média, mediana, erro padrão e porcentagem). Para verificar a existência de correlação entre as variáveis, primeiramente foi realizado 0 teste de Normalidade dos dados (Shapiro-wilk e Kolmogorov-smirnov), que demonstrou não haver normalidade dos dados, sendo por isso, escolhido o teste de correlação de Spearman. O número e a qualidade dos $\mathrm{CCO}$ em relação à fase do ciclo estral foram comparados pelo teste Kruskal-Wallis. Todas as análises foram feitas por meio da utilização do programa SPSS.versão 21.0.

Nas análises realizadas foram consideradas diferenças significativas quando $P<0,05$ e as correlações fortes quando $r \geq 0,60$.

\section{RESULTADOS E DISCUSSÃO}

A avaliação morfométrica dos ovários evidenciou que $100 \%$ apresentaram coloração rósea; $96,36 \%$ formato oval e $3,64 \%$ formato redondo; e $94,55 \%$ tinham consistência fibroelástica, enquanto $5,45 \%$ apresentaram consistência flácida.

Com relação ao formato dos ovários, o resultado apresentado está em concordância com a descrição feita por Ellenport (1986) e Frandson, Wilke e Fails (2005), os quais afirmaram que os ovários de cadelas possuem contorno alongado e oval e são achatados.

Não foram encontradas descrições sobre a coloração e a 
consistência de ovários caninos na literatura. Os resultados de consistência do presente estudo foram semelhantes aos descritos por Leal et al. (2013), para o qual a consistência dos ovários bubalino e bovino foi em sua maioria fibroelástica.

Nos testículos, a consistência pode refletir a sua funcionalidade ou alguma condição patológica. Testículos firmes estão relacionados à gametogênese normal e os flácidos podem indicar degeneração (Vale Filho, 1997). No presente estudo, apenas três cadelas apresentaram os ovários flácidos, sendo a taxa de recuperação média de CCOs de 21,3/animal, inferior a taxa de 30,35 CCOs/animal nas fêmeas com ovários fibroelásticos. Mais estudos são necessários para se estabelecer uma relação entre consistência e funcionalidade do ovário.

Os ovários pesaram, em média, 0,58 $\pm 0,08 \mathrm{~g}$ (mínimo de 0,1 e máximo de 3,06 g). Ellenport (1986) afirmou que os ovários caninos são pequenos, porém não descreveu valores numéricos para tal avaliação. Por outro lado, Frandson, Wilke e Fails (2005) citaram que os ovários caninos pesam cerca de 3 a $12 \mathrm{~g}$.

Quanto às dimensões ovarianas médias \pm erro padrão, constatou-se que o comprimento foi de $13,41 \pm 0,50 \mathrm{~mm}$ (mínimo de 8,70 e máximo de 27,69 $\mathrm{mm}$ ), altura de 6,46 $\pm 0,27 \mathrm{~mm}$ (mínima de 3,41 e máxima de $13,52 \mathrm{~mm})$ e largura de 7,81 $\pm 0,31 \mathrm{~mm}$ (mínima de 4,26 e máxima de $14,38 \mathrm{~mm}$ ).

Conforme verificado na tabela 2 , - peso médio dos ovários exibiu correlação com todas as dimensões ovarianas (comprimento: $r=0,86$; altura: $r=0,92$ e largura: $r=0,94 ; P<0,001)$. Isso já era esperado, pois quanto maior é o ovário, mais pesado ele deve ser.

Para Ellenport (1986), o comprimento médio do ovário da cadela é de $20 \mathrm{~mm}$, acima do encontrado no presente estudo. Para Frandson, Wilke e Fails (2005), os ovários caninos apresentam comprimento inferior a 2,5 $\mathrm{cm}$ e espessura inferior a $1,5 \mathrm{~cm}$.

A variação nas dimensões ovarianas descritas na literatura pode ser atribuída às diferenças raciais. No presente experimento, a maioria das cadelas eram SRD $(n=37)$ de baixo peso corporal e de raças pequenas como Poodle $(n=6)$ e Pinscher $(n=3)$.

$O$ peso do animal apresentou correlação positiva e significativa (Tabela 2) tanto com o peso do ovário $(r=0,76, \quad P<0,001)$ como com as dimensões ovarianas (comprimento: $r=$ 0,77; altura: $r=0,63$, e largura: $r=0,78$; $\mathrm{P}<0,001)$ permitindo concluir que 0 porte da fêmea interfere na biometria ovariana.

A fase do ciclo estral também é um fator a ser avaliado, visto que folículos ovarianos desenvolvidos (estro) e CLs (diestro) podem aumentar o peso e as medidas ovarianas. Tal consideração não foi verificada, pois não houve correlação significativa entre o estágio do ciclo estral e a biometria ovariana (Tabela 2). No entanto, acredita-se que aumentando o número de fêmeas avaliadas o efeito do ciclo pudesse ser comprovado estatisticamente.

O número médio \pm erro padrão de corpos lúteos encontrados foi de 3,62 \pm 0,49 (mínimo de zero e máximo de 15). O número de corpos lúteos apresentou correlação positiva com o peso $(r=0,72$; $\mathrm{P}<0,001)$, altura $(r=0,72 ; \mathrm{P}<0,001) \mathrm{e}$ largura $(r=0,69 ; P<0,001)$ do ovário (Tabela 2). O CL é formado por fibroblastos, células musculares lisas, do sistema imune, endoteliais e pela hiperplasia e/ou hipertrofia das células da granulosa e da teca interna (SALLES; ARAÚJO, 2010); por isso o diâmetro do CL maduro é maior do que do folículo ovulatório que o originou 
(HAFEZ; HAFEZ, 2004), ocupando um maior espaço no ovário.

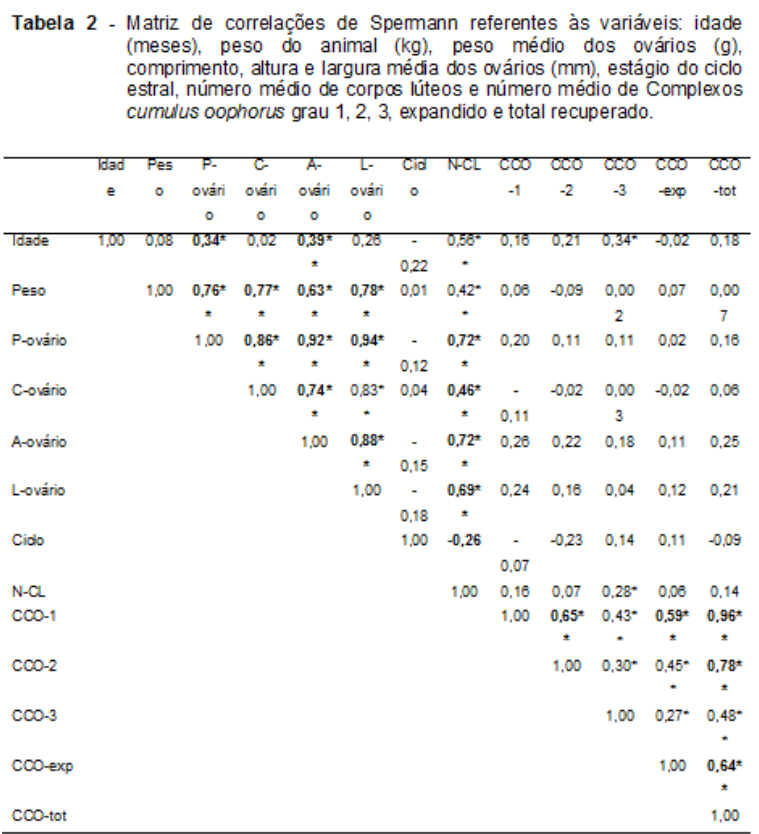

P- ovário: peso médio dos ovárics:

- ovár io: comprimento médio dos ovários;

A- ovário: attura média dos ovírios:

Ciclo-estágio do ciclo estral:

$N$ - CLL número médio de corpos lúteos:

CCO 1. número medio de Complexos cum uius cophons grau

CCO 2: número médio de Complexos cum uius cophons grau 2:

CCO exp: número médio de Complexos cum uhus ocohonss expandido; significativo $(P<0,05)$

Conforme se observa na tabela 2 , a idade das fêmeas não apresentou correlação com o número médio de CCOs de graus 1, 2 e 3 , expandido e total recuperado $(r<0,60 ; P>0,05)$. Tucholski et al. (2008) também não encontraram diferença significativa em relação à capacidade de doação de oócitos em fêmeas jovens e adultas.

Strom-Holst (2001) averiguou que a quantidade de CCOs recuperados é mais elevada em cadelas entre um e seis anos de idade, em comparação às cadelas de sete a 13 anos. Da mesma forma, Hewitt e England (1998) concluíram que quanto mais jovem é o animal, melhor é a qualidade dos CCOs recuperados. Rocha et al. (2006) recuperaram mais CCOs de cadelas senis (> 9 anos) do que jovens $(<2$ meses) e adultas (2 a 6,5 anos). No entanto, as cadelas senis proporcionaram menos CCOs de grau 1 e mais CCOs degenerados em relação às cadelas de outras idades.

$\mathrm{Na}$ literatura existem mais trabalhos evidenciando que as fêmeas de um a seis anos de idade são as mais indicadas como doadoras de oócitos (Strom-Holst, 2001; Rocha et al., 2006; Songsasen; Wildt, 2007) mas os próprios autores sugerem mais trabalhos confrontando os índices entre cadelas pré e pós-púberes.

No total foram recuperados 1642 CCOs, cuja média e erro padrão por animal foram de 29,85 \pm 4,20; dentre estes $58,16 \%(17,36 \pm 2,41)$ foram classificados como grau $1 ; 26,19 \%(7,82$ $\pm 1,50)$ grau $2 ; 3,78 \%(1,13 \pm 0,31)$ grau 3 e $11,88 \%(3,55 \pm 0,70)$ expandido. Adicionalmente, não foi possível a recuperação de $\mathrm{CCOs}$ de alguns animais, conforme pode ser observado na figura 1 .

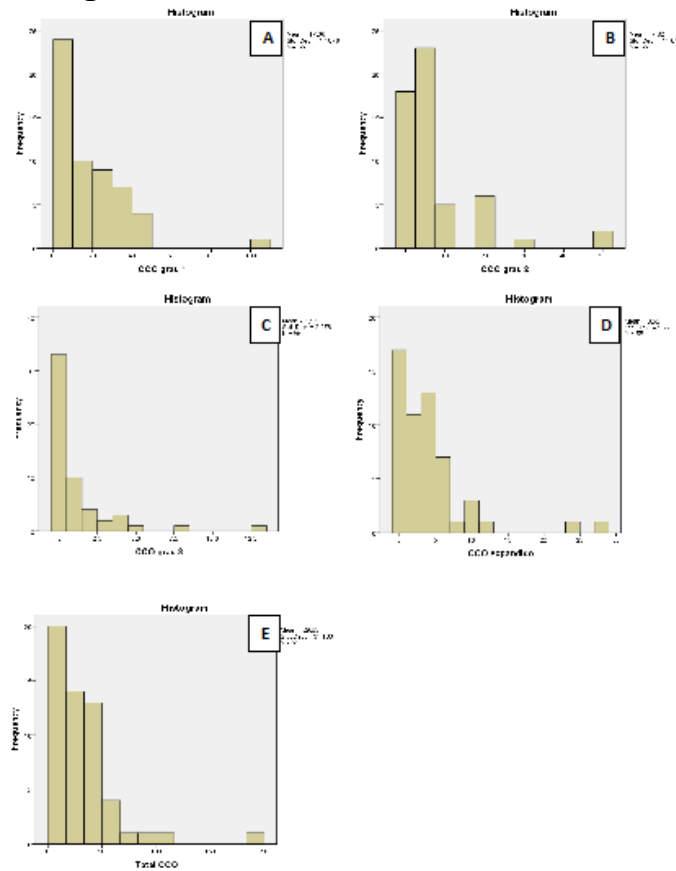

Figura 1 - Histogramas para a frequência de: A - CCO grau 1, B - CCO grau 2, C CCO grau 3, D - CCO expandido e E - número total de CCO recuperado.

Observou-se correlação positiva entre o número de CCOs grau $1 \mathrm{em}$ relação aos CCOs $2(r=0,65 ; P<0,001)$ e expandidos $(r=0,59 ; \quad P<0,001)$. Também se verificou correlação positiva entre $\mathrm{CCO}$ de grau $1(\mathrm{r}=0,96 ; \mathrm{P}<$ $0,001), \quad 2 \quad(r=0,78 ; \quad P<0,001) \quad e$ 
expandido $(r=0,64 ; \quad P<0,001)$ em relação ao número total de CCOs, pois estas estruturas foram as mais predominantes. No estudo de ApparícioFerreira (2010), em 49 cadelas, a recuperação de CCO grau I também foi superior às demais categorias. No entanto, no referido experimento, a recuperação média foi de 38 CCOs/animal, superior à encontrada neste estudo $(29,85)$.

Já no experimento de Tucholski et al. (2008), as taxas de recuperação de CCOs foram consideradas inadequadas nas fêmeas jovens (1 a 2,5 anos) e adultas (3 a 8 anos) avaliadas (12,6 e 7,1, respectivamente). Segundo estes autores, isso ocorreu devido ao baixo índice de escore corporal da população estudada. $\mathrm{Na}$ presente pesquisa, o escore de condição corporal não foi averiguado individualmente, mas foi procedida uma seleção prévia que impediu que animais mal nutridos ou doentes fossem submetidos à castração cirúrgica.

Das 55 cadelas estudadas, três $(5,5 \%)$ estavam no proestro, 23 $(41,82 \%)$ no estro, cinco $(9,09 \%)$ no diestro e $24(43,64 \%)$ no anestro. Para a determinação da fase do ciclo estral, foi necessário associar as informações do questionário aplicado aos proprietários com os resultados da dosagem de $\mathrm{P}_{4}$. Por se tratar de animais operados em campanhas de castração, na sua maioria, houve muita dificuldade em obter dados fidedignos, pois em muitas ocasiões não se sabia com exatidão a idade e a data do último episódio de estro.

Foi avaliada a influência da fase do ciclo estral na quantidade e qualidade dos CCOs recuperados, segundo resultados expressos na tabela 3.

\begin{tabular}{|c|c|c|c|c|}
\hline $\begin{array}{l}\text { Classificação } \\
\text { dos } \operatorname{CCOs}\end{array}$ & PROE STRO & ESTRO & DIE STRO & ANE STRO \\
\hline Grau 1 & 9 & 15 & 34 & 9 \\
\hline Grau 2 & 7 & 4 & 6 & 2,5 \\
\hline Grau 3 & 0 & 0 & 0 & 0,5 \\
\hline Expandido & 3 & 1 & 6 & 3 \\
\hline Total & 18 & 10 & 37 & 20,5 \\
\hline
\end{tabular}

No presente estudo, não foi constatada diferença estatística no número e na predominância dos diferentes graus de CCOs em relação à fase do ciclo estral. No entanto, Apparício-Ferreira (2010) observou que o número de oócitos recuperados e classificados de acordo com o aspecto morfológico em grau 1, 2 e 3 na fase folicular (proestro e estro) foi expressivamente superior às demais $(P<0,02)$.

Lopes et al. (2007) reportaram que tanto o número como a qualidade morfológica dos CCOs recuperados são influenciados pela idade do animal, mas não pela fase do ciclo estral (anestro ou diestro). Para Rodrigues e Rodrigues (2003) e Tucholski et al. (2008) a capacidade de doação de oócitos em fêmeas jovens e adultas também não foi alterada pelas fases estudadas (diestro e anestro).

A influência da fase do ciclo estral das cadelas e, consequentemente, dos eventos endócrinos pertinentes a cada período sobre a taxa de recuperação de oócitos ainda é controversa (APPARícIO et al., 2011). Parece que o estágio do ciclo estral é particularmente importante para a maturação dos oócitos in vitro (HEWIT; ENGLAND, 1997; MARTINS, 2005), porém não é unanimidade (APPARÍCIO-FERREIRA, 2006).

Os resultados contraditórios relatados na literatura acerca dos diversos aspectos avaliados no presente estudo reiteram a importância de novas pesquisas para se estabelecer padrões na espécie canina. 

cadelas em diferentes fases do ciclo estral

A figura 2 ilustra um CCO de grau 1 e outro de grau 2 recuperados no experimento.
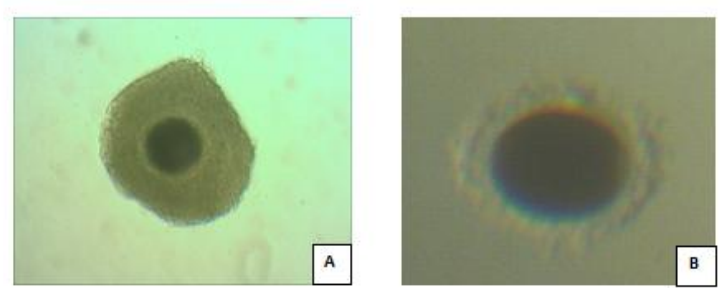

Figura 2- Imagens de CCOs em estereomicroscópios, com aumento de 40x. A: CCO grau 1 e B: CCO grau 2. Fonte:Binsfeld, 2013.

\section{CONCLUSÃO}

Nas condições experimentais deste estudo, conclui-se que os ovários caninos são predominantemente róseos, ovais e de consistência fibroelástica. A biometria ovariana foi influenciada pelo peso do animal e número de corpos lúteos. A fase do ciclo estral não influenciou o número total e a qualidade dos CCOs recuperados de ovários de cadelas.

\section{AGRADECIMENTOS}

A Universidade Paranaense Unipar pelo financiamento concedido a esta pesquisa.

\section{NOTAS INFORMATIVAS}

Este projeto foi aprovado pelo Comitê de Ética em Pesquisa Envolvendo Experimentação Animal da Universidade Paranaense - Unipar, sob protocolo 20986/2011.

\section{REFERÊNCIAS}

APPARÍCIO-FERREIRA, M. Efeito da suplementação de hCG, progesterona e estradiol na maturação nuclear e citoplasmática in vitro de oócitos de cadelas (Canis familiaris) obtidos por ovariosalpingohisterectomia. 65 f. 2006. Dissertação

(Mestrado em Cirurgia Veterinária) -
Universidade Estadual Paulista, Jaboticabal. 2006.

APPARÍCIO-FERREIRA, M. Maturação nuclear e citoplasmática de oócitos de cadelas colhidos em diferentes fases do ciclo estral e cultivados in vitro em meios sequenciais com hormônio e espermatozóides. 2010. Tese (Doutorado em Medicina Veterinária) Universidade Estadual Paulista, Jaboticabal. 2010.

APPARÍCIO, M.; MOSTACHIO, G.Q.; MOTHEO, T.F. et al. Maturação in vitro de oócitos caninos. Rev. Bras. Reprod. Anim., v.35, n.1, p.16-25, 2011.

BALL, L.; OTT, R.S.; MORTIMER, E.G.; SIMONS, J.C. Manual for breeding soundness examination of bulls. J. Soc. Theriogenol., v.12, p.1-65, 1983.

CHACÓN, J.; PÉREZ, Z.E.; MÜLLER, E. et al. Breeding soundness evaluation of extensively managed bulls in Costa Rica. Theriogenology, v.52, n.2, p.221-231, 1999.

CONCANNON, P. W., DIGREGORIO, G. B. Canine vaginal citology. In: BURKE, T.J. Small animal reproduction and infertility: a clinical approach to diagnosis and treatment.

Philadelphia: Lea \& Febiger, 1986. p.96-111.

CONCANNON, P.W.; HANSEL, W.; VISEK, W.J. The ovarian cycle on the bitch: plasma estrogen, $\mathrm{LH}$ and progesterone. Biology of

Reproduction, v.13, p.112-121, 1975.

DERIVAUX, J. Reprodução dos animais domésticos. Zaragoza: Acribia, 1980.

DYCE, K.M.; SACK, W.O.; WENSING, C.J.G. Tratado de anatomia veterinária. Rio de Janeiro: Elsevier, 2004.

ELLENPORT, C.R. Aparelho urogenital do carnívoro. In: GETTY, R. SISSON/GROSSMAN Anatomia dos animais domésticos. 5. ed. Rio de Janeiro: Guanabara Koogan, 1986.

ENGLAND, G.C.W. Allen's fertility and obstetrics in the dog. 2. ed. Oxford: WileyBlackwell, 1998.

FARSTAD, W. Assisted reproductive technology in canid species. Theriogenology, v.53, p.175186, 2000. 
FELDMAN, E. C.; NELSON, R. W. Canine and feline endocrinology and reproduction. 3 . ed. St. Louis: Saunders, 2004.

FRANDSON, R. D.; LEE WILKE, W.; FAILS, A.D. Anatomia e fisiologia dos animais de fazenda. 5. ed. Rio de Janeiro: Guanabara Koogan, 2005. p.432.

HAFEZ, E.S.E; HAFEZ, B. Reprodução animal. 7. ed., São Paulo: Manole, 2004.

HEWITT, D. A.: ENGLAND, G. C. W. The effect of preovulatory endocrine events upon maturation of oocytes of domestic bitch. Journal of Reproduction and Fertility, v.51, p.83-91, 1997.

HEWITT, D. A.: ENGLAND, G. C. W. The effect of oocyte size and bitch age upon oocyte nuclear maturation in vitro. Theriogenology, v. 49, n.5, p. 957-966, 1998.

JOCHLE, W.; ANDERSEN, A. C. The estrous cycle in the dog: a review. Theriogenology, v.7, n.3, p.113-140, 1977.

KÖNIG, H.E. Anatomia dos animais

domésticos. Porto Alegre: Artmed, 2004. p. 135-138.

LEAL, L.S.; MOYA-ARAÚJO, C.F.; OBA, E.; PRESTES, N.C. Morphometric characterization of bubaline and bovine ovaries at different phases of reproductive activity. Enciclopédia Biosfera, v.9, n.17, p.1929-1939, 2013.

LOPES, G. et al. Recovery rate, morphological quality and nuclear maturity of canine cumulusoocyte complexes collected from anestrous or diestrous bitches of different ages.

Theriogenology, v.68, n.9, p.821-825, 2007.

LUZ, M. R.; FREITAS, P. M. C.; PEREIRA, E. Z. Gestação e parto de cadelas: fisiologia, diagnóstico de gestação e tratamento das distocias. Revista Brasileira de Reprodução Animal, v.29, n.3/4, p.142-150, 2005.

MARTINS, L.R. Maturação nuclear de ovócitos de cadelas em estro e anestro submetidos à maturação in vitro. Dissertação (Mestrado em Reprodução Animal) - Faculdade de Medicina Veterinária e Zootecnia, Universidade Estadual Paulista, Botucatu, SP. 2005.

MUSOLINO, C.; GHIRELLI, C.O.; MORENO, L. M. Alterações do ciclo estral em cadelas.
2000. Disponível em:

<http://www.redevet.com.br>. Acesso em: 01 set. 2013.

OLIVEIRA, E. C. S.; MARQUES Jr., A. P.; NEVES, M. M. Endocrinologia reprodutiva e controle da fertilidade da cadela - revisão. Archives of Veterinary Science, v.8, n 1, p.112, 2003.

OLIVEIRA, E.C.S.; MARQUES Jr., A.P. Endocrinologia reprodutiva e controle da fertilidade da cadela. Rev. Bras. Reprod. Anim., v.30, n.1/2 p.11-18, 2006.

ROCHA, A.A.; BASTOS, R.; CUNHA, I.C.N.; ADONA, P.R.; SANTOS, J.A. Quantity and quality of oocytes recovered from donor bitches of different ages. Theriogenology, v.66, p.14651467, 2006.

RODRIGUES, B.A.; RODRIGUES, J.L. Influence of reproductive status on in vitro oocyte maturation in dogs. Theriogenology, v.60, p.5966, 2003.

SALLES, M.G.F.; ARAÚJO, A.A. Corpo lúteo cíclico e gestacional: revisão. Rev. Bras.

Reprod. Anim., v.34, n.3, p.185-194, 2010.

SONGSASEN, N.; WILDT, D.E. Oocyte biology and challenges in developing in vitro maturation systems in domestic dog. Anim. Reprod. Sci., v. 98, p. 2-22, 2007.

STROM-HOLST, B. Prediction of the oocytes recovery rate in the bitch. Journal of Veterinary Medicine, v.48, p.587-592, 2001.

TSUTSUI, T. Gamete physiology and timing of ovulation and fertilization in dogs. J. Reprod. Fertil., v.39, p.269-275, 1898.

TUCHOLSKI, A.P.; RASCHELLI, D.L.; MORAIS, R.N. et al. Quantidade de oócitos obtidos em fêmeas caninas em diferentes faixas etárias em diestro e anestro. Rev. Acad. Ciênc. Agrár. Ambient., v.6, n.3, p.341-347, 2008.

VALE FILHO, V.R. Andrologia no touro: avaliação genital, exame do sêmen e classificação por pontos. Rev. Bras. Reprod. Anim., v.21, n.3, p.7-13, 1997. 